Draft VERSION OCTOBER 1, 2020

Typeset using LATEX twocolumn style in AASTeX63

\title{
Detection of Pb II in the Ultraviolet Spectra of Three Metal-Poor Stars*
}

\author{
Ian U. Roederer, ${ }^{1,2}$ James E. Lawler, ${ }^{3}$ Erika M. Holmbeck, ${ }^{4,2}$ Timothy C. Beers,${ }^{5,2}$ Rana Ezzeddine, ${ }^{6,2}$ \\ Anna Frebel, ${ }^{7,2}$ Terese T. Hansen, ${ }^{8,9}$ Inese I. Ivans, ${ }^{10}$ Amanda I. Karakas, ${ }^{11,12}$ Vinicius M. Placco, ${ }^{13,2}$ And \\ Charli M. SAKARI ${ }^{14}$ \\ ${ }^{1}$ Department of Astronomy, University of Michigan, 1085 S. University Ave., Ann Arbor, MI 48109, USA \\ ${ }^{2}$ Joint Institute for Nuclear Astrophysics - Center for the Evolution of the Elements (JINA-CEE), USA \\ ${ }^{3}$ Department of Physics, University of Wisconsin-Madison, Madison, WI 53706, USA \\ ${ }^{4}$ Center for Computational Relativity and Gravitation, Rochester Institute of Technology, Rochester, NY 14623, USA \\ ${ }^{5}$ Department of Physics, University of Notre Dame, Notre Dame, IN 46556, USA \\ ${ }^{6}$ Department of Astronomy, University of Florida, Bryant Space Science Center, Gainesville, FL 32611, USA \\ ${ }^{7}$ Department of Physics and Kavli Institute for Astrophysics and Space Research, Massachusetts Institute of Technology, Cambridge, MA \\ 02139, USA \\ ${ }^{8}$ George P. and Cynthia Woods Mitchell Institute for Fundamental Physics and Astronomy, Texas AEM University, College Station, TX \\ 77843, USA \\ ${ }^{9}$ Department of Physics and Astronomy, Texas A $6 M$ University, College Station, TX 77843, USA \\ ${ }^{10}$ Department of Physics and Astronomy, University of Utah, Salt Lake City, UT 84112, USA \\ ${ }^{11}$ School of Physics and Astronomy, Monash University, VIC 3800, Australia \\ ${ }^{12}$ ARC Centre of Excellence for All Sky Astrophysics in 3 Dimensions (ASTRO 3D) \\ ${ }^{13}$ NSF's Optical-Infrared Astronomy Research Laboratory, Tucson, AZ 85719, USA \\ ${ }^{14}$ Department of Physics and Astronomy, San Francisco State University, San Francisco, CA 94132, USA
}

(Accepted for publication in the Astrophysical Journal Letters)

\begin{abstract}
We report the first detection of the $\mathrm{Pb}$ II line at $2203.534 \AA$ in three metal-poor stars, using ultraviolet spectra obtained with the Space Telescope Imaging Spectrograph on board the Hubble Space Telescope. We perform a standard abundance analysis assuming local thermodynamic equilibrium (LTE) to derive lead $(\mathrm{Pb}, Z=82)$ abundances. The $\mathrm{Pb}$ II line yields a higher abundance than $\mathrm{Pb}$ I lines by +0.36 \pm 0.34 dex and $+0.49 \pm 0.28$ dex in the stars HD 94028 and HD 196944, where $\mathrm{Pb}$ I lines had been detected previously. The $\mathrm{Pb}$ II line is likely formed in LTE, and these offsets affirm previous calculations showing that $\mathrm{Pb}$ I lines commonly used as abundance indicators underestimate the $\mathrm{Pb}$ abundance in LTE. $\mathrm{Pb}$ is enhanced in the $s$-process-enriched stars $\mathrm{HD} 94028([\mathrm{~Pb} / \mathrm{Fe}]=+0.95 \pm 0.14)$ and HD $196944([\mathrm{~Pb} / \mathrm{Fe}]=+2.28 \pm 0.23)$, and we show that ${ }^{208} \mathrm{~Pb}$ is the dominant $\mathrm{Pb}$ isotope in these two stars. The $\log \varepsilon(\mathrm{Pb} / \mathrm{Eu})$ ratio in the $r$-process-enhanced star HD 222925 is $0.76 \pm 0.14$, which matches the Solar System $r$-process ratio and indicates that the Solar System $r$-process residuals for $\mathrm{Pb}$ are, in aggregate, correct. The $\mathrm{Th} / \mathrm{Pb}$ chronometer in HD 222925 yields an age of $8.2 \pm 5.8 \mathrm{Gyr}$, and we highlight the potential of the $\mathrm{Th} / \mathrm{Pb}$ chronometer as a relatively model-insensitive age indicator in $r$-process-enhanced stars.
\end{abstract}

Keywords: Nucleosynthesis (1131); R-process (1324); S-process (1419); Stellar abundances (1577); Ultraviolet astronomy (1736)

Email: iur@umich.edu

* Based on observations made with the NASA/ESA Hubble Space Telescope, obtained at the Space Telescope Science Institute (STScI), which is operated by the Association of Universities for Research in Astronomy, Inc. (AURA) under NASA contract NAS 5-26555. These observations are associated with programs GO-14161, GO-14765, and GO-15657. This paper includes data taken at The McDonald Observatory of The University of Texas at Austin.

\section{INTRODUCTION}

The element lead ( $\mathrm{Pb}, Z=82)$ has fascinating nucleosynthetic origins. Three of the four stable $\mathrm{Pb}$ isotopes $\left({ }^{206} \mathrm{~Pb},{ }^{207} \mathrm{~Pb}\right.$, and $\left.{ }^{208} \mathrm{~Pb}\right)$ and the one stable bismuth (Bi, $Z=83$ ) isotope $\left({ }^{209} \mathrm{Bi}\right)$ serve as both the highmass termination point of the slow $n$-capture process ( $s$-process) and the low-mass termination point of ac- 
tinide $\alpha$-decay chains of radioactive isotopes produced in the rapid $n$-capture process ( $r$-process).

$\mathrm{Pb}$ and $\mathrm{Bi}$ accumulate during the $s$-process as $n$-capture and $\alpha$-decay reactions cycle indefinitely (e.g., Burbidge et al. 1957). Clayton \& Rassbach (1967) recognized that most $\mathrm{Pb}$ in the Solar System could not form through the $r$-process or a "smooth extension of the [s-process] circumstances attending the synthesis for $A<200$." Gallino et al. (1998) and Travaglio et al. (2001) showed that the high neutron-to-seed ratio that occurs in low-metallicity stars during the asymptotic giant branch (AGB) phase of evolution is responsible. Most of that $\mathrm{Pb}$ is expected to be ${ }^{208} \mathrm{~Pb}$, which sits at both the $Z=82$ proton shell closure and the $N=126$ neutron shell closure, and its $n$-capture cross section is nearly an order of magnitude smaller than that of neighboring nuclei (cf. Ratzel et al. 2004). Observations confirm enhanced $\mathrm{Pb}$ and $\mathrm{Bi}$ abundances, relative to lighter $s$-process elements, in many $s$-process-enriched metalpoor stars (e.g., Van Eck et al. 2001; Aoki et al. 2002; Ivans et al. 2005).

$\mathrm{Pb}$ is also a remarkable element in $r$-process nucleosynthesis because it is the final decay product for most isotopes heavier than ${ }^{209} \mathrm{Bi}$, including the three longlived isotopes of the actinides thorium (Th, $Z=90$ ) and uranium (U, $Z=92):{ }^{232} \mathrm{Th},{ }^{235} \mathrm{U}$, and ${ }^{238} \mathrm{U}$, which decay to ${ }^{208} \mathrm{~Pb},{ }^{207} \mathrm{~Pb}$, and ${ }^{206} \mathrm{~Pb}$, respectively. The actinide elements can only be produced by $r$-process nucleosynthesis, yet our understanding of actinide production remains incomplete. $\mathrm{Pb}$ abundances in metal-poor stars can bridge this gap in our understanding. More than $85 \%$ of $\mathrm{Pb}$ in old, metal-poor stars enhanced in $r$-process elements is formed through the decay of radioactive nuclei with $A>209$ (Cowan et al. 1999), so $\mathrm{Pb}$ abundances provide an important constraint on the production of these isotopes (e.g., Schatz et al. 2002; Wanajo et al. 2002; Eichler et al. 2019).

A few studies (e.g., Plez et al. 2004) have attempted to constrain model predictions by assessing the $r$-process contribution to $\mathrm{Pb}$ abundances, but that work has been limited by observational uncertainties. Only a few lines of $\mathrm{Pb}$ I are detectable in the optical spectrum. The most commonly-used line, at $4057.807 \AA$, is often weak and blended. Furthermore, $\mathrm{Pb}$ is mostly ( $\gtrsim 99 \%$ ) ionized in the atmospheres of metal-poor stars, and departures from local thermodynamic equilibrium (LTE) impact the $\mathrm{Pb}$ abundances derived from $\mathrm{Pb}$ I lines (Mashonkina et al. 2012). These observational challenges can be overcome by detecting $\mathrm{Pb}$ in its dominant ionization state, singly-ionized $\mathrm{Pb}$.

In this Letter, we examine new and archival ultraviolet (UV) spectra of three metal-poor stars taken with the Space Telescope Imaging Spectrograph (STIS) on board the Hubble Space Telescope. These spectra show the Pb II line at 2203.534 $\AA$, which is the only Pb II transition accessible in near-UV, optical, or near-infrared spectra of late-type stars. This line has been observed previously in the spectra of a few chemically-peculiar Atype stars (e.g., Faraggiana 1989; Cowley et al. 2016). Previous attempts (Roederer et al. 2014b) to detect the $\mathrm{Pb}$ II line in STIS E230M spectra $(R \equiv \lambda / \Delta \lambda=30,000)$ of metal-poor stars have been unsuccessful. Here, we present the first detection of this $\mathrm{Pb}$ II line in metalpoor stars.

\section{OBSERVATIONS AND STELLAR SAMPLE}

STIS spectra of only three metal-poor stars yield compelling detections of the $\mathrm{Pb}$ II line at 2203.534 $\AA$. These stars were selected for observations over the years because they are bright, metal-poor, and in two cases show extreme enhancements of $n$-capture elements. UV spectra obtained with STIS (Woodgate et al. 1998) cover the $\mathrm{Pb}$ II line at $2203 \AA$ with the high resolving power $(R$ $=114,000)$ of the E230H grating, as summarized in Table 1. Archival observations of the star HD 94028 were downloaded from the Mikulski Archive for Space Telescopes (MAST). Two new sets of observations of the stars HD 196944 and HD 222925 were also downloaded through the MAST and processed automatically by the CALSTIS software package. All spectra were shifted to a common velocity, co-added, and continuum normalized using IRAF. The signal-to-noise $(\mathrm{S} / \mathrm{N})$ ratios per pixel in the co-added spectra are listed in Table 1 . These modest $\mathrm{S} / \mathrm{N}$ ratios are sufficient to detect the lines of interest because of the high resolving power.

HD 94028 shows moderate levels of enhancement of both $r$-process and $s$-process elements (Roederer et al. 2016; Peterson et al. 2020; see Table 1). Roederer et al. also found evidence that an intermediate $n$-capture process ( $i$-process) may contribute to some of the $Z<56$ elements in HD 94028. That study derived the $\mathrm{Pb}$ abundance from one $\mathrm{Pb}$ I line at 2833.054 $\AA$ and concluded that the $s$-process dominates the origin of the $\mathrm{Pb}$ in HD 94028. There is no evidence from radial velocity (RV) measurements that HD 94028 is in a binary system.

HD 196944 is one of the original "Pb stars" identified by Van Eck et al. (2001). Numerous studies over the years have confirmed that HD 196944 is a carbon-enhanced metal-poor star enhanced in elements produced by the $s$-process (CEMP-s star; e.g., Začs et al. 1998; Placco et al. 2015). The Pb abundance in HD 196944 has been derived previously from $\mathrm{Pb}$ I lines at 2833 and $4057 \AA$. HD 196944 exhibits RV variations, and Placco et al. calculated an orbital period of 1325 $\pm 12 \mathrm{~d}$.

HD 222925 is a member of the class of highly $r$-process-enhanced, or $r$-II, stars (as defined in Holmbeck et al. 2020). All heavy elements in HD 222925 were produced via $r$-process nucleosynthesis (Roederer et al. 2018a). $\mathrm{Pb}$ had not been detected previously in HD 222925, but Roederer et al. placed a tight upper limit on the $\mathrm{Pb}$ abundance from the $\mathrm{Pb}$ I line at 
Table 1. Log of Observations, Model Atmosphere Parameters, Metallicities, $\mathrm{Ba}, \mathrm{Eu}$, and $\mathrm{Pb}$ Abundances

\begin{tabular}{cccc}
\hline \hline Quantity & HD 94028 & HD 196944 & HD 222925 \\
\hline Prog. ID & GO-14161 & GO-14765 & GO-15657 \\
PI & Peterson & Roederer & Roederer \\
Data Sets & OCTKB0010-6030 & OD5A01010-14010 & ODX901010-60030 \\
$V_{\text {mag }}$ & 8.22 & 8.40 & 9.03 \\
$\mathrm{~S} / \mathrm{N} @ 2200 \mathrm{~A}$ & $50 / 1$ & $40 / 1$ & $30 / 1$ \\
$T_{\text {eff }}(\mathrm{K})$ & $6087 \pm 84(1)$ & $5170 \pm 100(2)$ & $5636 \pm 103(3)$ \\
$\log g[\mathrm{cgs}]$ & $4.37 \pm 0.13(1)$ & $1.60 \pm 0.25(2)$ & $2.54 \pm 0.17(3)$ \\
$v_{\mathrm{t}}\left(\mathrm{km} \mathrm{s}{ }^{-1}\right)$ & $1.10 \pm 0.20(1)$ & $1.55 \pm 0.10(2)$ & $2.20 \pm 0.20(3)$ \\
$V_{t}\left(\mathrm{~km} \mathrm{~s}{ }^{-1}\right)$ & $1.6 \pm 0.3(4)$ & $6.8 \pm 0.5(5)$ & $7.0 \pm 0.5(6)$ \\
{$[\mathrm{M} / \mathrm{H}]$} & $-1.60 \pm 0.10(1)$ & $-2.41 \pm 0.25(2)$ & $-1.50 \pm 0.10(3)$ \\
{$[\mathrm{Fe} / \mathrm{H}]$} & $-1.65 \pm 0.08(1)$ & $-2.41 \pm 0.18(2)$ & $-1.46 \pm 0.08(6)$ \\
$\log \varepsilon(\mathrm{Ba})$ & $1.06 \pm 0.11(4)$ & $1.00 \pm 0.11(2)$ & $1.26 \pm 0.09(3)$ \\
$\log \varepsilon(\mathrm{Eu})$ & $-0.62 \pm 0.13(4)$ & $-2.00 \pm 0.10(2)$ & $0.38 \pm 0.09(3)$ \\
$\log \varepsilon(\mathrm{Pb})$ & $1.34 \pm 0.12(4)$ & $1.91 \pm 0.30(4)$ & $1.14 \pm 0.16(4)$ \\
{$[\mathrm{Ba} / \mathrm{Fe}]$} & $+0.53 \pm 0.09(4)$ & $+1.23 \pm 0.26(2)$ & $+0.54 \pm 0.06(6)$ \\
{$[\mathrm{Eu} / \mathrm{Fe}]$} & $+0.51 \pm 0.13(4)$ & $-0.11 \pm 0.10(2)$ & $+1.32 \pm 0.08(6)$ \\
{$[\mathrm{Pb} / \mathrm{Fe}]$} & $+0.95 \pm 0.14(4)$ & $+2.28 \pm 0.23(4)$ & $+0.56 \pm 0.14(4)$ \\
\hline
\end{tabular}

Note-We have rederived the barium (Ba, $Z=56 ; 3$ optical Ba II lines), europium (Eu, $Z=63 ; 2$ optical Eu II lines), and $\mathrm{Pb}$ ( 1 UV $\mathrm{Pb}$ I line) abundances in HD 94028 using our adopted model atmosphere and a high-resolution optical spectrum obtained using the Tull Coudé spectrograph on the $2.7 \mathrm{~m}$ Harlan J. Smith Telescope at McDonald Observatory (see Roederer et al. 2014a for details) These model parameters and the $\mathrm{Ba}$ and $\mathrm{Eu} \log \varepsilon$ abundances are in agreement with those derived by Peterson et al. (2020). References are indicated by the numbers in parentheses: (1) Roederer et al. (2018b); (2) Placco et al. (2015); (3) Roederer et al. (2018a); (4) This study; (5) Roederer et al. (2008); (6) Roederer et al. (in preparation)

$4057 \AA$. HD 222925 shows no evidence of RV variations that would signal a stellar companion.

\section{ANALYSIS}

\subsection{Atomic Data}

There are four stable isotopes of $\mathrm{Pb}:{ }^{204} \mathrm{~Pb}(1.4 \%$ in Solar System material), ${ }^{206} \mathrm{~Pb}(24.1 \%),{ }^{207} \mathrm{~Pb}(22.1 \%)$, and ${ }^{208} \mathrm{~Pb}(52.4 \%)$. Of these isotopes, ${ }^{207} \mathrm{~Pb}$ has nonzero nuclear spin, $I=1 / 2$, and thus has hyperfine splitting (HFS) structure. The field shift, which results from the volume difference between nuclei with the same number of protons but different numbers of neutrons, also creates isotope shifts (IS). We adopt the ground level and excited level HFS $A$ values and the IS measurements from Bouazza et al. (1986) for the $\mathrm{Pb}$ II line at $2203 \AA$. We adopt the atomic transition probability from Quinet et al. (2007), $\log (g f)=-0.24 \pm 0.07$, because it is normalized to a radiative lifetime measurement from laser induced fluorescence. For comparison, the National Institutes of Standards and Technology (NIST) Atomic Spectral Database (ASD; Kramida et al. 2019) recommends $\log (g f)=-0.14$, based on theoretical calculations referenced there, which is in fair agreement with our adopted value. We present the line component pattern in Table 2.

\subsection{Model Atmospheres}

We adopt the model parameters (effective temperature, $T_{\text {eff }}$; log of the surface gravity, $\log g$; microturbulent velocity, $v_{\mathrm{t}}$; model metallicity, $\left.[\mathrm{M} / \mathrm{H}]\right)$ derived previously for these three stars, for consistency. HD 94028 is a main sequence dwarf, while HD 196944 and HD 222925 are red horizontal branch stars. We interpolate model atmospheres from the 1D, $\alpha$-enhanced ATLAS9 grid of models (Castelli \& Kurucz 2004). Our synthetic spectra also include a macroturbulent velocity $\left(V_{t}\right)$ component, which improves the fits to the high-resolution E230H spectra. We derive $V_{t}$ by fitting the observed profiles of isolated lines of Fe-group elements. These values are listed in Table 1.

\section{3. $\mathrm{Pb}$ Abundances}

Figure 1 illustrates the $\mathrm{Pb}$ II line in the spectrum of each of the three stars. Continuum regions around this line are easily identified, and they are matched by the synthetic spectra. We are confident that the absorption at $2203.534 \AA$ is due to $\mathrm{Pb}$ II for several reasons. The line strength varies with the expected heavy-element abundances in these stars, not the abundances of iron-group elements that are responsible for most UV absorption lines. This line is - by many orders of magnitude - the strongest $\mathrm{Pb}$ II line with $\lambda>2000 \AA$, so there is no expectation that other $\mathrm{Pb}$ II lines could be detectable. Furthermore, no other plausible species are found at this wavelength in the NIST ASD or the Kurucz (2011) line lists. Unidentified lines at 2203.427 and $2203.645 \AA$ could be explained by Co II and V I transitions, respectively, only if the $\log (g f)$ values recommended by the NIST ASD or the Kurucz (2011) linelists are underestimated by several dex. We treat their strengths as free parameters in our analysis, and this choice does not influence the derived $\mathrm{Pb}$ abundances.

The $\mathrm{Pb}$ II line is on the linear part of the curve-ofgrowth in HD 94028 and HD 222925, but it is saturated in HD 196944. We derive abundances using the 2017 version of the LTE line analysis software MOOG (Sneden 1973; Sobeck et al. 2011). We adopt an s-process mix of $\mathrm{Pb}$ isotopes (Sneden et al. 2008) for HD 94028 and HD 196944 and an $r$-process mix for HD 222925. We generate the line list based on Kurucz (2011), Peterson et al. (2017), and the NIST ASD. We match the synthetic spectra to the observed spectra following the general methods described by Roederer et al. (2012).

Table 1 lists the derived abundances. The $\mathrm{Pb}$ abundance is defined as $\log \varepsilon(\mathrm{Pb}) \equiv \log _{10}\left(N_{\mathrm{Pb}} / N_{\mathrm{H}}\right)+12.0$. The abundance ratio of elements $\mathrm{Pb}$ and $\mathrm{Fe}$ relative to the Solar ratio is defined as $[\mathrm{Pb} / \mathrm{Fe}] \equiv$ $\log _{10}\left(N_{\mathrm{Pb}} / N_{\mathrm{Fe}}\right)-\log _{10}\left(N_{\mathrm{Pb}} / N_{\mathrm{Fe}}\right) \odot$, where $\log \varepsilon(\mathrm{Pb})_{\odot}=$ 2.04 and $\log \varepsilon(\mathrm{Fe})_{\odot}=7.50$. Following Roederer et al. (2018a), we compute $1 \sigma$ uncertainties by drawing $10^{3}$ resamples of the stellar parameters, $\log (g f)$ values, and equivalent widths approximated from the abundance 
Table 2. Hyperfine Structure and Isotope Shifts for the Pb II Line at $2203 \AA$

\begin{tabular}{cccccccc}
\hline $\begin{array}{c}\text { Wavenumber } \\
\left(\mathrm{cm}^{-1}\right)\end{array}$ & $\begin{array}{c}\lambda_{\text {air }} \\
(\AA)\end{array}$ & $F_{\text {upper }}$ & $F_{\text {lower }}$ & $\begin{array}{c}\text { Component Position } \\
\left(\mathrm{cm}^{-1}\right)\end{array}$ & $\begin{array}{c}\text { Component Position } \\
(\AA)\end{array}$ & $\begin{array}{c}\text { Strength } \\
\text { Isotope }\end{array}$ \\
\hline 45367.486 & 2203.5342 & 0.5 & 1.5 & -0.175014 & 0.008501 & 1.000 & 204 \\
45367.486 & 2203.5342 & 0.5 & 1.5 & -0.067014 & 0.003255 & 1.000 & 206 \\
45367.486 & 2203.5342 & 1.0 & 2.0 & 0.061236 & -0.002975 & 0.625 & 207 \\
45367.486 & 2203.5342 & 1.0 & 1.0 & 0.095236 & -0.004626 & 0.125 & 207 \\
45367.486 & 2203.5342 & 0.0 & 1.0 & -0.252764 & 0.012278 & 0.250 & 207 \\
45367.486 & 2203.5342 & 0.5 & 1.5 & 0.040986 & -0.001991 & 1.000 & 208 \\
\hline
\end{tabular}

Note-Energy levels from the NIST ASD and the index of air are used to compute the center-of-gravity wavenumbers and air wavelengths, $\lambda_{\text {air }}$, for a Solar System isotopic composition (Meija et al. 2016). Line component positions are given relative to those values. The strengths of each component are easily adjustable using Table 2 because a Solar System abundance pattern has not been assumed, and strengths are normalized to sum to 1 for each isotope. For example, the $\log (g f)$ value of the ${ }^{207} \mathrm{~Pb}$ component with $F_{\text {upper }}=F_{\text {lower }}=1.0$ in a Solar System mix with $f_{207}=0.221$ would be $\log _{10}\left(0.221 \times 0.125 \times 10^{-0.24}\right)=-1.80$. Table 2 is available in the online edition of the journal in machine-readable format.

derived via synthesis using a reverse curve-of-growth method.

\subsection{The Pb Isotope Mix}

The $\mathrm{Pb}$ isotope mix has not been assessed previously in any metal-poor star. The HFS of the ${ }^{207} \mathrm{~Pb}$ isotope, particularly the upper level of the line at $2203 \AA$, and the IS of the four $\mathrm{Pb}$ isotopes are wide compared to the width of the stellar line profiles shown in Figure 2. As the isotope mix shifts from the $r$-process, where the ${ }^{206} \mathrm{~Pb}$ isotope $(35.9 \%)$ and wide HFS of the ${ }^{207} \mathrm{~Pb}$ isotope $(45.1 \%)$ are expected to dominate, to an $s$-process mix, where the ${ }^{208} \mathrm{~Pb}$ isotope is expected to dominate $(69.5 \%)$, the absorption line profile narrows and shifts to shorter wavelengths.

The observed $\mathrm{Pb}$ II line profiles in HD 94028 and HD 196944 both favor a narrower profile, suggesting that an even- $A$ isotope dominates. We evaluate which isotope it might be by comparing the line centroid to the isotope wavelengths. We set the local wavelength zeropoint of the observed spectrum relative to the synthetic spectra using three relatively unblended Fe II lines, with wavelengths known to better than $0.0011 \AA$ (Nave \& Johansson 2013), located in the same echelle order that contributes most of the signal to the $\mathrm{Pb}$ II line. We also account for uncertainty in the center-of-gravity wavelength of the $\mathrm{Pb}$ II line (0.0007 $\AA$; Wood et al. 1974) and measurement uncertainties in the $\mathrm{Fe}$ II and $\mathrm{Pb}$ II line centroids. As shown in Figure 2, the centroid of the observed Pb II line in HD 94028 and HD 196944 favors absorption by the isotopes situated farthest to the blue, ${ }^{207} \mathrm{~Pb}$ and ${ }^{208} \mathrm{~Pb}$. Our method of setting the wavelength zeropoint using Fe II lines appears to have produced a small mismatch between the observed and synthetic line profiles in HD 94028 and HD 222925. Manual adjustment $(\leq 1 \sigma)$ of the observed spectrum in each case so that it matches the blue side of the line, which is relatively insensitive to the $\mathrm{Pb}$ isotope mix, still favors the ${ }^{207} \mathrm{~Pb}$ and ${ }^{208} \mathrm{~Pb}$ isotopes.

We conclude from these two tests - the narrow line profiles and the positions of the line centroids - that the ${ }^{208} \mathrm{~Pb}$ isotope is dominant in HD 94028 and HD 196944. The $\mathrm{S} / \mathrm{N}$ is too low to draw any conclusions about HD 222925.

\section{DISCUSSION}

\subsection{Neutral Pb and Non-LTE Effects}

The abundances derived from the $\mathrm{Pb}$ II line are higher than the abundances or upper limits derived from the $\mathrm{Pb}$ I lines in all three stars: $[\mathrm{Pb} \mathrm{II} / \mathrm{Pb} \mathrm{I}]=+0.36 \pm 0.34$, $+0.49 \pm 0.28$, and $>+0.04$ in HD 94028, HD 196944, and HD 222925, respectively. Mashonkina et al. (2012) computed non-LTE corrections to the LTE abundances for several metal-poor atmospheres. That study found that the lower levels of the Pb I lines at 2833 and $4057 \AA$ experience similar deviations from LTE. The ground state of singly-ionized $\mathrm{Pb}$ is well-described by LTE in their calculations. We assume that the excited $6 s^{2} 6 p$ level that gives rise to the $\mathrm{Pb}$ II line at $2203 \AA$ is also well-described by LTE. The Mashonkina et al. non-LTE corrections for red giants (dwarfs) range from +0.26 to +0.62 dex $(+0.22$ to +0.32 dex $)$ when using a Drawin scaling factor, $S_{H}$, of 0.1 , which relates to the strength of inelastic collisions with neutral hydrogen. No models in their grid exactly match the stars in our sample, but the closest models predict non-LTE corrections $\approx+0.27$ and +0.52 dex for HD 94028 and HD 196944, respectively, which match the offsets we derive in LTE. We support 

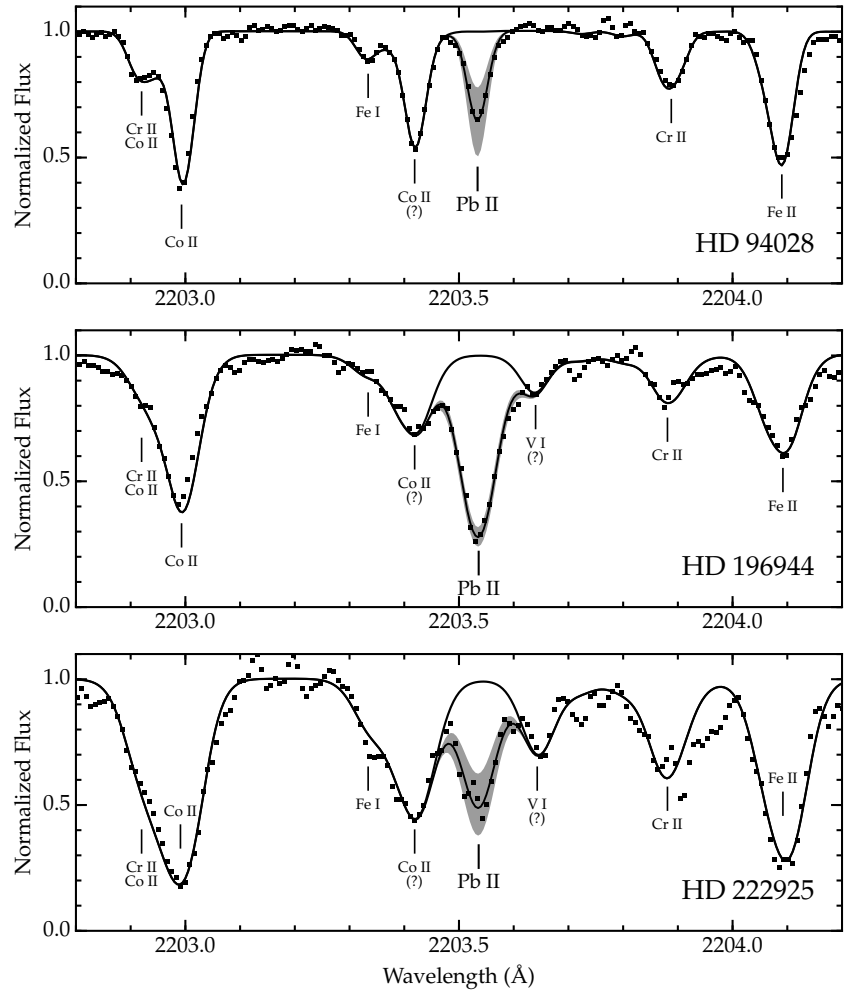

Figure 1. Sections of the STIS/E230H spectra of HD 94028, HD 196944, and HD 222925 around the $\mathrm{Pb}$ II line at $2203 \AA$. The filled dots represent the observed spectrum. The solid lines represent a synthetic spectrum with the best-fit abundance in each star, and the gray bands represent a change in this abundance by a factor of \pm 2 (0.3 dex). The solid black line with no gray bands represents a synthetic spectrum with no contributions from $\mathrm{Pb}$. Other line identifications are marked.

the conclusion of Mashonkina et al. that departures from LTE impact $\mathrm{Pb}$ abundances derived from $\mathrm{Pb}$ I lines.

$\mathrm{Pb}$ is often used to constrain the $s$-process or $i$-process models used to explain nucleosynthesis patterns in stars (e.g., Hampel et al. 2019). A change in $[\mathrm{Pb} / \mathrm{Fe}]$ by +0.4 dex is significant and could affect the final neutron exposure inferred from models, which sets, for example, the estimated timescale for an $i$-process event. Future work should incorporate non-LTE corrections to abundances derived from $\mathrm{Pb}$ I lines or derive $\mathrm{Pb}$ abundances in LTE directly from the UV Pb II line.

\section{2. $\mathrm{Pb}$ in the s-process}

Our LTE results confirm the enhanced $\mathrm{Pb}$ abundances in the s-process-enhanced stars HD 94028 and HD 196944. We derive $[\mathrm{Pb} / \mathrm{Ba}]=+1.05 \pm 0.35$ in HD 196944. This is in good agreement with the AGB $s$-process model of Bisterzo et al. (2010) discussed at length in Placco et al. (2015) (see also Abate et al. 2015). We derive $[\mathrm{Pb} / \mathrm{Ba}]=+0.42 \pm 0.17$ in HD 94028, which
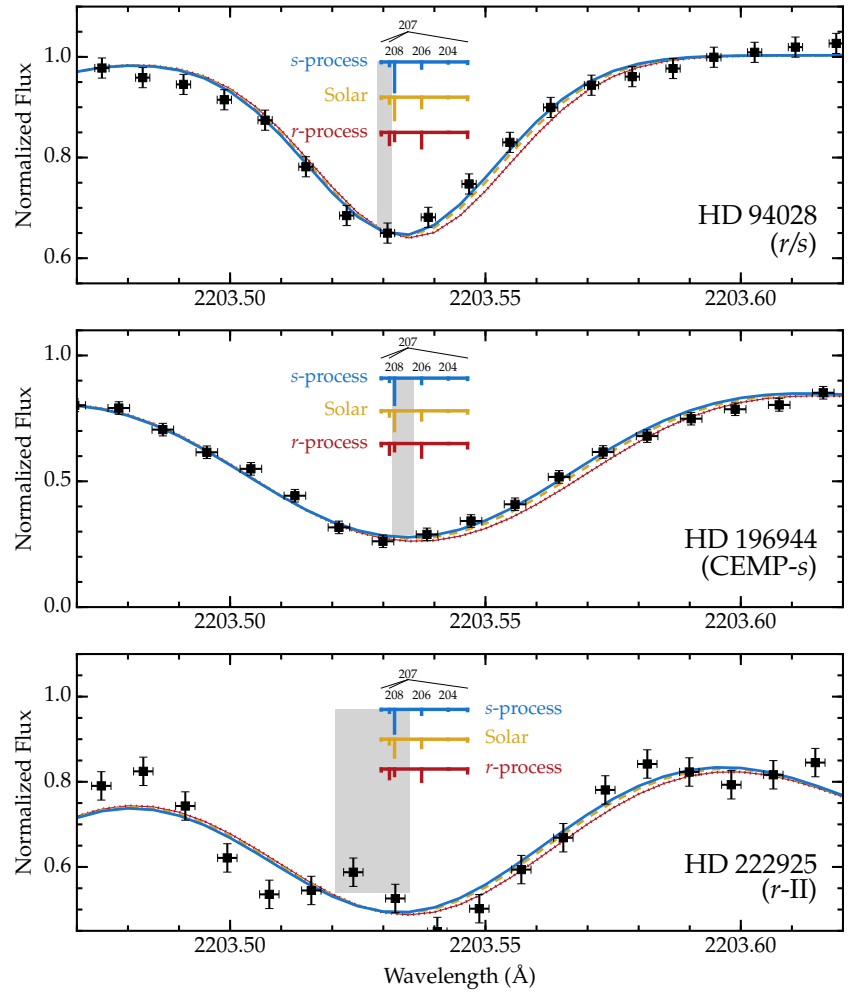

Figure 2. Tight zoom around the $\mathrm{Pb}$ II line. The $\mathrm{Pb}$ abundance is held fixed and the isotope mixes are varied. The blue solid line represents an $s$-process isotope mix $\left(f_{204} / f_{206} / f_{207} / f_{208}=0.025 / 0.143 / 0.137 / 0.695\right)$ from Sneden et al. (2008), the yellow dashed line represents the Solar isotope $\operatorname{mix}(0.014 / 0.241 / 0.221 / 0.524)$ (Meija et al. 2016), and the red studded line represents an $r$-process isotope mix $(0.000 / 0.359 / 0.451 / 0.190)$ from Sneden et al. The filled dots represent the observed spectrum. The shaded gray box in each panel represents the $\pm 1 \sigma$ line centroid of the $\mathrm{Pb}$ II line in the observed spectrum. The isotope mixes inferred by our analysis (Section 3.4) are insensitive to the initial isotope mixes assumed for each star (Section 3.3) once the abundances have been fixed.

supports the interpretation of Roederer et al. (2016) that the $\mathrm{Ba}$ and $\mathrm{Pb}$ in $\mathrm{HD} 94028$ originated mainly via the $s$-process. Our isotopic analysis reaffirms theoretical predictions that the large $\mathrm{Pb}$ overabundances in low-metallicity $s$-process environments are dominated by ${ }^{208} \mathrm{~Pb}$.

\section{3. $\mathrm{Pb}$ in the r-process}

The $\log \varepsilon(\mathrm{Pb} / \mathrm{Eu})$ ratio in $\mathrm{HD} 222925,0.76 \pm 0.14$, matches the Solar System $r$-process ratio, $\log \varepsilon(\mathrm{Pb} / \mathrm{Eu})$ $=0.76 \pm 0.10$ (Sneden et al. 2008; Bisterzo et al. 2014; Prantzos et al. 2020). This result indicates that the $r$-process residuals for the $\mathrm{Pb}$ isotopes are, in aggregate, correct when the effects of low-metallicity AGB stars (i.e., the so-called "strong component") are included. 
Clayton \& Rassbach (1967) argued that the dominant $r$-process isotopes of $\mathrm{Pb}$ must be ${ }^{206} \mathrm{~Pb}$ and ${ }^{207} \mathrm{~Pb}$. The $\mathrm{Pb}$ II line centroid in HD 222925 is not in conflict with this reasoning, although the $\mathrm{S} / \mathrm{N}$ is too low in our spectrum to support a more definitive statement.

The close coupling between $\mathrm{Th}$ and $\mathrm{Pb}$ enables the use of $\mathrm{Th} / \mathrm{Pb}$ as a chronometer pair that is relatively insensitive to the details of the $r$-process model used to calculate the initial production ratio. HD 222925 does not exhibit a prominent actinide boost (Roederer et al. $2018 \mathrm{a})$, and its $\log \varepsilon(\mathrm{Th} / \mathrm{Pb})$ ratio is $-1.20 \pm 0.14$. The $\log \varepsilon(\mathrm{Th} / \mathrm{Pb})$ ratio in HD 222925 corresponds to an age of $8.2 \pm 5.8$ Gyr using the production ratios from Roederer et al. (2009). The permitted age range is large, but improving the $\mathrm{S} / \mathrm{N}$ at the $\mathrm{Pb}$ II line in future observations would improve the age precision. $\mathrm{Pb}$ lines are easier to detect in metal-poor stars than the U II line at $3859 \AA$, and the $\mathrm{Th} / \mathrm{Pb}$ chronometer offers an alternative model-insensitive age indicator to the $\mathrm{U} / \mathrm{Th}$ chronometer in $r$-process-enhanced stars.

\section{ACKNOWLEDGMENTS}

We thank E.A. Den Hartog for useful discussions and the referee for a quick and helpful report. I.U.R., J.E.L., T.C.B., A.F., and V.M.P. acknowledge support pro- vided by NASA through grants GO-14765 and GO15657 from STScI, which is operated by the AURA under NASA contract NAS5-26555. I.U.R., T.C.B., R.E., A.F., E.M.H., and V.M.P. acknowledge financial support from grant PHY 14-30152 (Physics Frontier Center/JINA-CEE) awarded by the U.S. National Science Foundation (NSF). We acknowledge additional support from NSF grants AST-1716251 (A.F.) and AST1815403 (I.U.R.). T.T.H. acknowledges generous support from the George P. and Cynthia Woods Institute for Fundamental Physics and Astronomy at Texas A\&M University. Parts of this research were supported by the Australian Research Council Discovery Project scheme (DP170100521) and Centre of Excellence for All Sky Astrophysics in 3 Dimensions (ASTRO 3D), through project number CE170100013. This research has made use of NASA's Astrophysics Data System Bibliographic Services; the arXiv pre-print server operated by Cornell University; the SIMBAD and VizieR databases hosted by the Strasbourg Astronomical Data Center; the ASD hosted by NIST; the MAST at STScI; and the Image Reduction and Analysis Facility (IRAF) software packages.

\section{Facility: HST (STIS), Smith (Tull Coudé)}

Software: IRAF (Tody 1993), matplotlib (Hunter 2007), MOOG (Sneden 1973), numpy (van der Walt et al. 2011), R (R Core Team 2013)

\section{REFERENCES}

Abate, C., Pols, O. R., Izzard, R. G., \& Karakas, A. I. 2015, A\&A, 581, A22

Aoki, W., Ryan, S. G., Norris, J. E., et al. 2002, ApJ, 580, 1149

Bisterzo, S., Gallino, R., Straniero, O., Cristallo, S., \& Käppeler, F. 2010, MNRAS, 404, 1529

Bisterzo, S., Travaglio, C., Gallino, R., Wiescher, M., \& Käppeler, F. 2014, ApJ, 787, 10

Bouazza, S., Guern, Y., \& Bauche, J. 1986, Journal of Physics B Atomic Molecular Physics, 19, 1881

Burbidge, E. M., Burbidge, G. R., Fowler, W. A., \& Hoyle, F. 1957, Reviews of Modern Physics, 29, 547

Castelli, F., \& Kurucz, R. L. 2004, ArXiv e-prints. https://arxiv.org/abs/astro-ph/0405087

Clayton, D. D., \& Rassbach, M. E. 1967, ApJ, 148, 69

Cowan, J. J., Pfeiffer, B., Kratz, K.-L., et al. 1999, ApJ, 521,194

Cowley, C. R., Ayres, T. R., Castelli, F., et al. 2016, ApJ, 826,158

Eichler, M., Sayar, W., Arcones, A., \& Rauscher, T. 2019, ApJ, 879, 47

Faraggiana, R. 1989, A\&A, 224, 162
Gallino, R., Arlandini, C., Busso, M., et al. 1998, ApJ, 497, 388

Hampel, M., Karakas, A. I., Stancliffe, R. J., Meyer, B. S., \& Lugaro, M. 2019, ApJ, 887, 11

Holmbeck, E. M., Hansen, T. T., Beers, T. C., et al. 2020, ApJS, 249, 30

Hunter, J. D. 2007, Computing in Science and Engineering, 9,90

Ivans, I. I., Sneden, C., Gallino, R., Cowan, J. J., \& Preston, G. W. 2005, ApJL, 627, L145

Kramida, A., Ralchenko, Y., Reader, J., \& NIST ASD

Team. 2019, NIST Atomic Spectra Database (ver. 5.7.1), [Online]. Available: https://physics.nist.gov/asd, National Institute of Standards and Technology, Gaithersburg, MD.

Kurucz, R. L. 2011, Canadian Journal of Physics, 89, 417

Mashonkina, L., Ryabtsev, A., \& Frebel, A. 2012, A\&A, 540, A98

Meija, J., Coplen, M. B., Berglund, M., et al. 2016, Pure and Applied Chemistry, 88, 293

Nave, G., \& Johansson, S. 2013, ApJS, 204, 1

Peterson, R. C., Barbuy, B., \& Spite, M. 2020, A\&A, 638, A64 
Peterson, R. C., Kurucz, R. L., \& Ayres, T. R. 2017, ApJS, 229, 23

Placco, V. M., Beers, T. C., Ivans, I. I., et al. 2015, ApJ, 812,109

Plez, B., Hill, V., Cayrel, R., et al. 2004, A\&A, 428, L9

Prantzos, N., Abia, C., Cristallo, S., Limongi, M., \& Chieffi, A. 2020, MNRAS, 491, 1832

Quinet, P., Biémont, E., Palmeri, P., \& Xu, H. L. 2007, Journal of Physics B Atomic Molecular Physics, 40, 1705

R Core Team. 2013, R: A Language and Environment for Statistical Computing, R Foundation for Statistical Computing, Vienna, Austria. http://www.R-project.org/

Ratzel, U., Arlandini, C., Käppeler, F., et al. 2004, PhRvC, 70, 065803

Roederer, I. U., Karakas, A. I., Pignatari, M., \& Herwig, F. 2016, ApJ, 821, 37

Roederer, I. U., Kratz, K.-L., Frebel, A., et al. 2009, ApJ, 698, 1963

Roederer, I. U., Lawler, J. E., Sneden, C., et al. 2008, ApJ, 675,723

Roederer, I. U., Preston, G. W., Thompson, I. B., et al. 2014a, AJ, 147, 136

Roederer, I. U., Sakari, C. M., Placco, V. M., et al. 2018a, ApJ, 865, 129

Roederer, I. U., Sneden, C., Lawler, J. E., et al. 2018b, ApJ, 860, 125

Roederer, I. U., Lawler, J. E., Sobeck, J. S., et al. 2012, ApJS, 203, 27

Roederer, I. U., Schatz, H., Lawler, J. E., et al. 2014b, ApJ, 791,32
Schatz, H., Toenjes, R., Pfeiffer, B., et al. 2002, ApJ, 579, 626

Sneden, C., Cowan, J. J., \& Gallino, R. 2008, ARA\&A, 46, 241

Sneden, C. A. 1973, PhD thesis, The University of Texas at Austin.

Sobeck, J. S., Kraft, R. P., Sneden, C., et al. 2011, AJ, 141, 175

Tody, D. 1993, in Astronomical Society of the Pacific Conference Series, Vol. 52, Astronomical Data Analysis Software and Systems II, ed. R. J. Hanisch, R. J. V. Brissenden, \& J. Barnes, 173

Travaglio, C., Gallino, R., Busso, M., \& Gratton, R. 2001, ApJ, 549, 346

van der Walt, S., Colbert, S. C., \& Varoquaux, G. 2011, Computing in Science Engineering, 13, 22

Van Eck, S., Goriely, S., Jorissen, A., \& Plez, B. 2001, Nature, 412, 793

Wanajo, S., Itoh, N., Ishimaru, Y., Nozawa, S., \& Beers, T. C. 2002, ApJ, 577, 853

Wood, D. R., Ross, C. B., Scholl, P. S., \& Hoke, M. L. 1974, Journal of the Optical Society of America (1917-1983), 64, 1159

Woodgate, B. E., Kimble, R. A., Bowers, C. W., et al. 1998, PASP, 110, 1183

Začs, L., Nissen, P. E., \& Schuster, W. J. 1998, A\&A, 337, 216 